\title{
DEM simulations of the particle flow on a centrifugal fertilizer spreader
}

\author{
P. Van Liedekerke ${ }^{\text {a,* }}$, E. Tijskens ${ }^{\text {a,* }}$, E. Dintwa ${ }^{a}$, F. Rioual ${ }^{\text {b }}$, J. Vangeyte ${ }^{c}$, H. Ramon $^{\text {a }}$ \\ a Department BIOSYST, K.U. Leuven, Kasteelpark Arenberg 30, B-3001 Leuven, Belgium \\ b CEMAGREF Clermont-Ferrand, Montoldre sur Allier, Domaine des Palaquins, 03150 Montoldre, France \\ c ILVO, Burg. Van Gansberghelaan 115 bus, 9820 Merelbeke, Belgium
}

\section{A R T I C L E I N F O}

\section{Article history:}

Received 3 July 2007

Received in revised form 6 August 2008

Accepted 7 August 2008

Available online $\mathrm{xxxx}$

\section{Keywords:}

Discrete element method

Granular fertilizer

Centrifugal spreader

Multi particle

\begin{abstract}
A B S T R A C T
Usually, the performance of centrifugal spreaders must be evaluated in large halls by capturing the fertilizer distribution patterns in standardized tests, often carrying a big cost to the manufacturers. In contrast, this paper proposes a first attempt to model a particle flow subjected to a spinning disc using the Discrete Element Method (DEM) starting from the particle outflow of a bin, using flat as well as inclined discs. The model is validated by experiments in two different ways. The first manner is the measurement of the cylindrical mass distribution along the edge of the disc by a device that collects the fertilizer particles in a tray of baskets around the disc. A second method consists of collecting the particles on the ground after their ballistic flight through the air. Both validation methods are relatively cheap and fit into the present statistical or qualitative interpretation of DEM simulations. Additionally, a number of rotational disc speeds is chosen (300-650 rpm) to incorporate velocity dependent effects of the particle flow. It was found that the DEM simulations show a good qualitative and considerable quantitative agreement with the experiments. The deviations between the simulations and experiments are profound at high disc rotational speeds (500$650 \mathrm{rpm}$ ) and can be identified as (1) an underestimation of the simulated particle velocities at the edge of the disc and (2) a too low dispersion on the (vertical) simulated particle velocities at the edge of the disc. A parameter study revealed that (1) can be resolved by introducing a velocity dependent friction coefficient, in agreement with literature. The influence of other model parameters such as particle damping and stiffness appears to be small, while the introduction of a rolling friction coefficient to mimic rolling resistance or particle shape does not provide any answer either, and hence reason (2) at this moment must be addressed to unknown external factors such as disc plane vibrations appearing at higher disc speeds.
\end{abstract}

(c) 2008 Elsevier B.V. All rights reserved.

\section{Introduction}

For the past few decades there has been a growing concern about the environmental implications associated with the use of soil fertilizers. In Europe, over 90\% of fertilizer is distributed using spinning disc spreaders. The popularity of spinning disc spreaders lies with their relative low cost and high accuracy. However, the performance of these machines is highly dependent on the particle properties (e.g. friction coefficients, particle shape...). This results in large differences in spread patterns depending on the fertilizer type as well as the prevailing weather conditions (e.g. air humidity) [8].

The primary concern of a spreader manufactures is the construction of spreading tables which link fertilizer properties and doses $(\mathrm{kg} / \mathrm{ha})$ to the machine settings. This can be either done by measuring the spread pattern in spreader halls (expensive, even though nowadays there are techniques which are less time consuming [16], or to interpolate the spreading characteristics from data bases or characteristics which are

\footnotetext{
* Corresponding authors.

E-mail addresses: Paul.VanLiedekerke@biw.kuleuven.be (P. Van Liedekerke), Engelbert.Tijskens@biw.kuleuven.be (E. Tijskens).
}

easy to measure (mostly inaccurate). Despite their need, these tests are 50 expensive, and require huge amounts of fertilizer. Furthermore, they 51 are not uniquely standardized [14].

Reumers et al. [18] proposed a method based on measurements 53 with a cylindrical collector tray around the disc edge, and photo- 54 graphic tracking for determining particle velocities. By using a ballistic 55 model, the total spread pattern can be determined. To apply the same 56 principle online in the field, determination of particle velocities 57 methods have been proposed by Vangeyte et al. [23], Grift and Hofstee 58 [6]. The primary objective of these models is to replace the expensive 59 spread hall measurements.

The granular flow on a spinning disc with vanes is similar to paddle 61 systems (mixers), which have been studied by Steward et al. [21], Zhou 62 et al. [26], and Kuo et al. [12] using DEM simulations. In these 63 experiments, particles are located within the boundary of a cylinder. 64 Using the Positron emission particle tracking (PEPT) technique, an 65 individual tracer particle in the flow can be monitored instantly and 66 non-invasively. DEM simulations can be validated, although based on 67 a single particle. Experiments with a granular flow on a centrifugal 68 spreader are usually performed by collecting the particles after they 69 leave the disc, resulting in a spatial spread pattern. Although these 70 


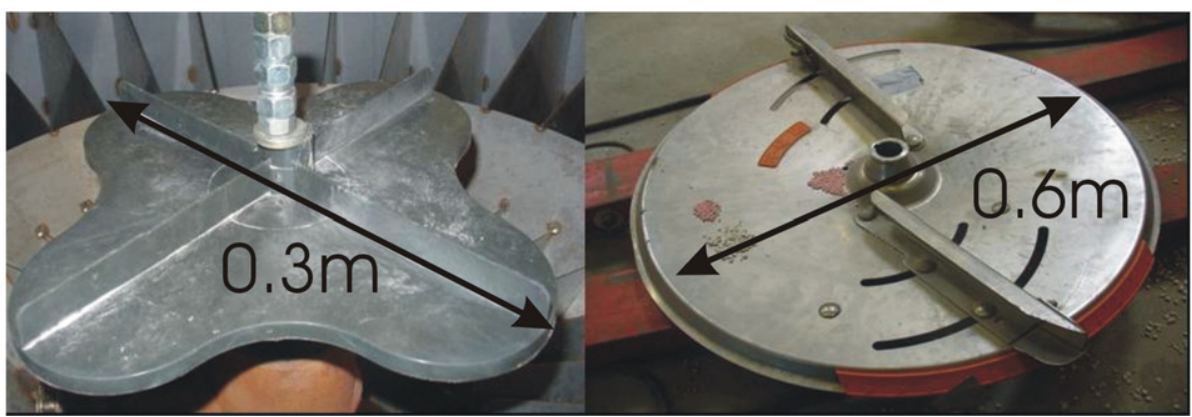

Fig. 1. Disc types used; left: lawn spreader disc (type A); right field spreader disc (type B).

experiments provide no information on what happens during the process, they can accurately summarize an experiment and hence be a suitable partner for DEM validations. In this paper, an experimental set up of a particle flow on a spinning disc is given in detail, as well as the comparison with DEM simulations.

This paper has the intention to demonstrate that the discrete element method has the potential to be an even more cost efficient alternative to obtain the spreading pattern for a fertilizer. Therefore, a series of simulations and experiments with matching conditions was conducted. Although the results of the comparison are quantified, they still must be interpreted in a statistical and qualitative way, as with most present-day DEM- simulations. Nevertheless, they can provide the manufacturer valuable information about the particle flow on a spinning disc.

\subsection{The discrete element model}

In DEM, the contact deformation between two bodies is represented as a single vector describing the deformation of the contact point in the normal and tangential directions. This vector is related to contact forces by a contact force model. For the problem at hand, Van Liedekerke et al. [24] have proposed a Hertz-Kuwabara-Kono model $[13,2,20,17]$ for the normal contact force, $\mathbf{N}$ :

$\mathbf{N}=\min \left(0,-\sqrt{\left|\delta_{N}\right|}\left(k_{N} \delta_{N}+c_{N} \dot{\delta}_{N}\right)\right) \mathbf{n}$

Here, $k_{N}$ and $c_{N}$ are the non-linear contact stiffness and damping parameter, respectively. The quantity $\delta_{N}$ is termed the virtual overlap (deformation) of the contacting bodies. The vector $\mathbf{n}$ is a unit vector normal to the contact surface. For the tangential contact force, T, a linear elastic spring is augmented with dry Coulomb friction, which, 98 formulated as an incremental expression, reads:

$\mathrm{T}_{t+\Delta t}=-\min \left(\left\|\mathbf{Q}_{t}^{t+\Delta t} \mathbf{T}_{t}+k_{T} \Delta \boldsymbol{\delta}_{T}\right\|, \mu|| \mathbf{N}_{t+\Delta t} \|\right) \mathbf{t}$

Here, the quantity $\Delta \boldsymbol{\delta}_{T}$ is the incremental equivalent of $\delta_{N}$ in the 100 normal contact force model over the time step $\Delta t$. For a detailed 103 account of how $\Delta \boldsymbol{\delta}_{T}$ is computed, see [24]. In Eq. (2) $\mathbf{Q}_{t}^{t+\Delta t}$ is a rotation 104 matrix which maps the orientation of the particle at time step $t$ to that 105 at time step $t+\Delta t$ and accounts for the rotation of the contact plane 106 and the tangential contact force during the time step. In Eq. (2) the 107 min-operator implements the Coulomb friction limit with constant 108 friction coefficient $\mu$ between two bodies in contact:

$\|\mathbf{T}\| \leq \mu\|\mathbf{N}\|$,

Although more realistic and complex tangential force models exist 111 [19], the choice for this simple is justified if the collisions are mainly 113 driven by friction, and the use of a complex tangential model is less 114 necessary. By simple linear reasoning, such a condition is equivalent to 115 the exceeding of the friction limit at the beginning of contact:

$\Delta \delta_{T} k_{T} \geq \mu k_{N} \Delta \delta_{N}$

Or, by terms of contact velocities, and making abstraction of both 118 normal and tangential stiffness:

$\frac{\dot{\delta}_{T}}{\dot{\delta}_{N}} \geq \mu$

Eq. (5) reads that such a process should be driven by shear 123 collisions, and is more feasible for lower friction coefficients. It could 124 be argued that the particle flow on a spinning disc fulfils this 125
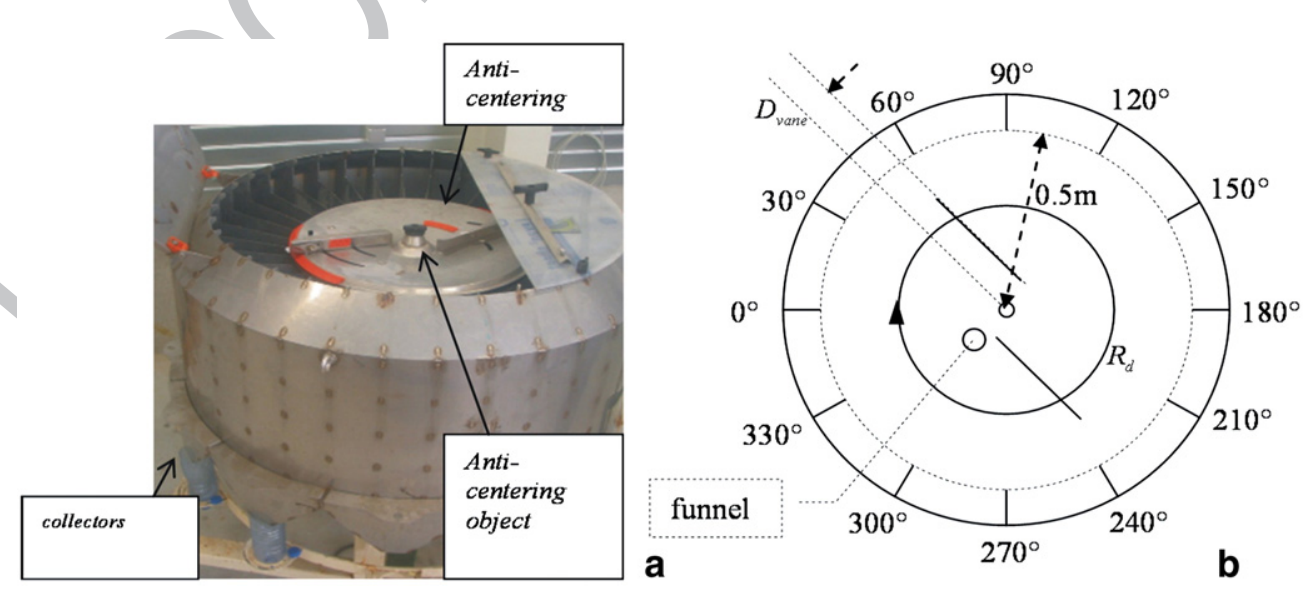

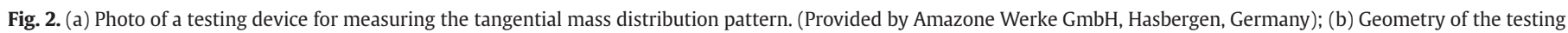
device for measuring the tangential mass distribution pattern (top view). 
condition, because the particles are mainly driven outwards the disc centre, with little difference in normal speeds, while due to the angular velocities and contact with the vanes, the shear velocities are substantial. Hence, a tangential force model as proposed in Eq. (2) was implemented. However, in the case of static a process, the choice of such simple model may not be appropriate.

The use of a rolling friction coefficient to incorporate the resistance to rolling of spherical particles as proposed by Brilliantov et al. [3] or Zhou et al. [25] was primarily not considered in the model. Even for a nearly spherical fertilizer particle, it is hard to determine such a coefficient in a rolling experiment, because of irregularities on its surface. A rough estimation could be obtained by means of its restitution coefficient [3]. However, the use of a rolling friction coefficient could have significant consequences on the results via the complex rolling-sliding regime of the particles and therefore a brief parameter study is discussed in Section 4.2.

The contact forces, together with gravity, drive the motion of the particles, which is described by Newton's and Euler's equations of motion for each particle in the system [1,7]. The equations of motion are solved with a Leapfrog integration method [5].

\section{Experimental methods}

Testing the spreadability of new kinds of fertilizer spreaders can be done in 2 ways; one possibility is to use a device like the one shown in Fig. 2, which surrounds the disk with a circular collector tray. The method is fast and cheap, but provides only information about the tangential mass distribution, and not about the velocities of the particles leaving the disk. Optical measurement techniques can bring a solution here $[6,18,23]$.

A second method lies with the direct measurement of the total spread pattern produced by the spinning disc in a (large) hall. This however requires a considerable amount of fertilizer particles in order to get a reliable distribution. Furthermore, it is a very time consuming activity, although as previously mentioned, nowadays faster techniques are available. Both kinds of experiments were applied in this paper; the details are discussed below.

The particles used in the experiments are NPK granules with a fairly spherical shape. Their size distribution can be assumed as lognormal. A sample of 100 particles was monitored to determine the radii and density.

The experiments were conducted with two kinds of discs. The first one (Type A) is a disc for a domestic lawn spreader (see Fig. 1, left). A

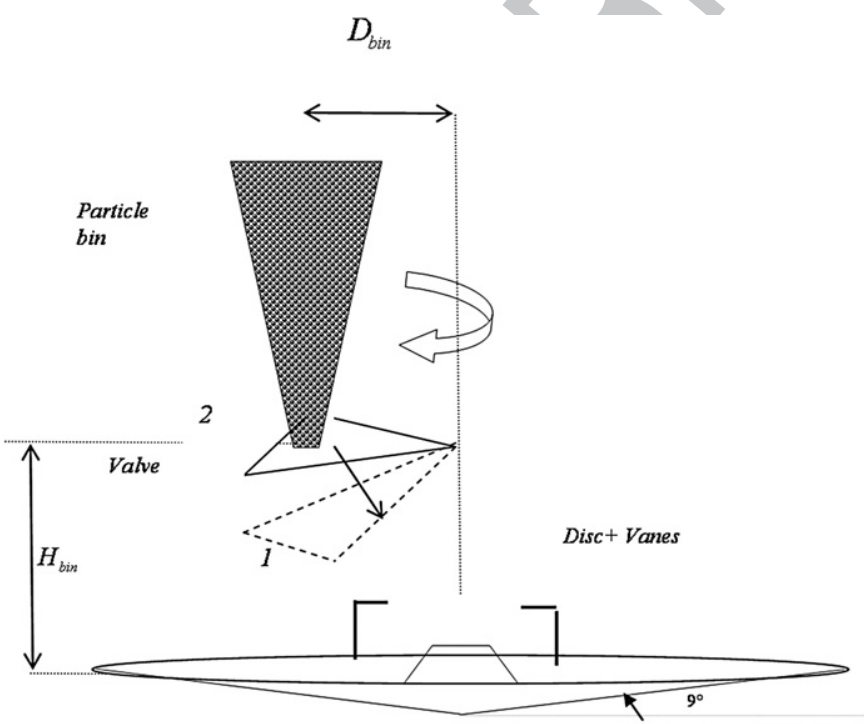

Fig. 3. Side view of the disc and particle bin.

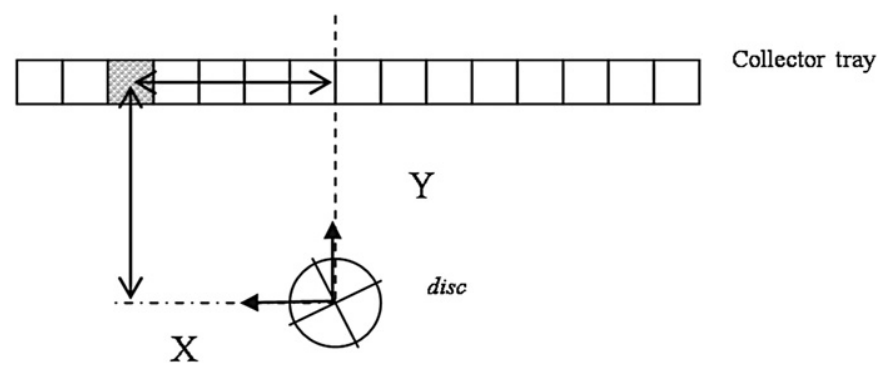

Fig. 4. Scheme of the TSP experimental set up.

lawn spreader typically features a 4-vane disc, usually made of a light 167 material (e.g. PVC). The size of the disc is small, e.g. $0.15 \mathrm{~m}$ radius. To 168 reduce the production cost, the disc is deformed in the regions 169 between the vanes, and the vanes themselves have no horizontal flaps 170 which normally keep the particles lower than the vane height. This 171 kind of a spreader is made predominantly for domestic use.

172

The second disc (Type B) has a conical inclination $\vartheta_{d}$ of $9^{\circ}$ and a 173 radius $R_{d}$ of $0.29 \mathrm{~m}$. In the experiment, two L-shaped vanes (see Fig. 1, 174 right), of length $L_{\mathrm{vane}}=0.3 \mathrm{~m}$ and height $H_{\mathrm{vane}}=0.036 \mathrm{~m}$ were used. As 175 with most current spreaders, the vanes are not placed radially, but 176 have an offset $D_{\text {vane }}=0.045 \mathrm{~m}$ relative to the centre of the disc. This 177 kind of spreader is made mainly for exhaustive field applications. 178

\subsection{Cylindrical measurements}

179

The cylindrical spread pattern (CSP) is measured by a device (also 180 called Carrousel) where the spinning disc is surrounded by a 181 cylindrical collector tray in which the particles are captured. The 182 disc is driven by a $380 \mathrm{~V}$ electrical motor with accurate control of rpm 183 $(+/-1 \mathrm{rpm})$. In Fig. 2a, such apparatus (provided by Amazone Werke) is 184 shown. The collectors span sectors of $30^{\circ}$ and are positioned at a 185 distance of $0.5 \mathrm{~m}$ from the centre of the disk.

186

In order to prevent the particles from moving inwards, to the 187 centre of the conical disk, a metal body which has the shape of a 188 truncated cone, nearly cylindrical, is mounted in the middle of the disc 189 (see Figs. 2a and 3). To avoid spill over of particles, most current 190 spreaders have vanes with horizontal flaps to keep the particles below 191 the vane height as much as possible.

192

The fertilizer particles are fed on to the disc by a plastic funnel with 193 an orifice diameter of $0.029 \mathrm{~m}$ using a valve which is mounted above 194 the spreader. The funnel is placed at a height $H_{\text {bin }}=0.11 \mathrm{~m}$ above the 195 disk, a distance $D_{\text {bin }}=0.1 \mathrm{~m}$ from the centre of the disk and an angular 196 position of $0^{\circ}$.

In each experiment, the funnel was filled with (NPK) fertilizer for 198 domestic use. The particles are assumed to be distributed log-normally 199 in size with a measured average diameter of $0.00126 \mathrm{~m}$ and a standard 200 deviation of $0.00014 \mathrm{~m}$. The measured material ${ }^{1}$ density of the 201 fertilizer is $1775 \mathrm{~kg} / \mathrm{m}^{3}$. The amount of particles in each experiment 202 is $0.5 \mathrm{~kg}$. As the funnel yields a mass flow of $0.11 \mathrm{~kg} / \mathrm{s}$, the funnel is 203 emptied in $4.55 \mathrm{~s}$. Thus, at $300 \mathrm{rpm}$ it takes 45 vane passages to empty 204 under the funnel, and 90 times at $600 \mathrm{rpm}$. The particle flow is 205 initialized by manually opening a valve (see Fig. 3), after which the 206 particles fall onto the disk and are taken by the vanes to be thrown in 207 the collectors. The contents of the collectors are then weighed.

\subsection{Rectangular measurements}

In a rectangular experiment, the transverse static pattern (TSP) is 210 measured. This is done by aligning baskets at a distance vector $(\mathbf{X}, \mathbf{Y}, \mathbf{Z}) 211$ from the disc centre (see Fig. 4), where $\mathbf{X}$ is the transverse distance, $\mathbf{Y} 212$ is the longitudinal distance, and $\mathbf{Z}$ is the height between the baskets 213

\footnotetext{
${ }^{1}$ Not bulk density.
} 


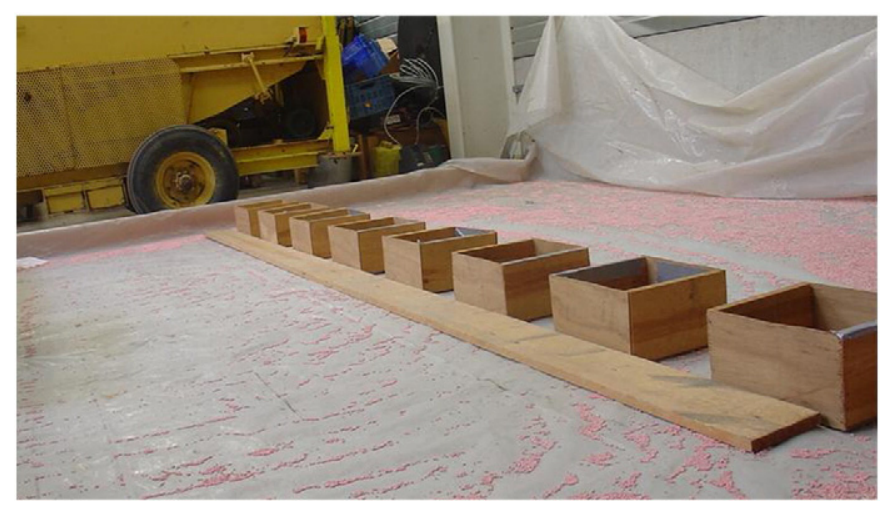

Fig. 5. Lined collector tray baskets for measuring the transverse spread pattern.

and the disc. By measuring the TSP over different $\mathbf{Y}$ distances (when the tray is shifted incrementally over the total $\mathbf{Y}$ range), a total transverse spread pattern (TTSP) can be obtained. The disc is driven by a $1 \mathrm{~kW}$ electrical motor, and the speed is monitored by an rpm counter before each experiment.

The relative height $\mathbf{Z}$ of the disc to the baskets was $0.28 \mathrm{~m}$ and it was kept constant over all experiments. The baskets have dimensions of $0.25 \mathrm{~m} \times 0.25 \mathrm{~m}$. The inside of the baskets is covered with a soft material to prevent particles from jumping out when impacting at high speed. The total length of the tray is $3.5 \mathrm{~m}$, equivalent to 16 baskets (see Fig. 5).

\section{Computational setup}

Ideally, the simulations should respect the initial conditions and the geometry of the experiment. However, there are some situations where simplifications cannot be avoided. As examples, first, the NPK particles are by production not perfect spheres. Their diameter varies depending on the direction along which it is measured and the also show some surface roughness. In the simulation, the particles are approximated as perfect spheres, and the surface roughness is neglected. Secondly, the particle assembly in the simulation is a random realisation of spherical particles according to the above mentioned particle size distribution and their initial positions are determined by a simulation in which they are dropped into the bin with the valve closed. These initial conditions have been used for all simulations. This is a simplification of the real situation where the particle assembly would be completely random. Lastly, the positions of the vanes at $t=0$, when the valve is opened, is not known. However, this uncertainty is averaged by the number of vane passages in each experiment, and the number of experiments.

\subsection{Model parameter settings}

Interaction parameters between particles are hard to determine, especially for such small objects as fertilizer grains. Basic methods [24] were used to obtain useful values for contact stiffness, damping and friction properties for impact between a particle and a metal plate. As the experimental determination of particle-particle collision forces for fertilizer particles is not practically feasible, some simple assumptions are introduced to derive particle-particle contact force model parameters from particle-metal plate experiments. Treating the elastic and dissipative part of Eq. (1) independently, then the elastic part is the Hertz' law for 2 spheres which is given by:

$N=\frac{4 \sqrt{R_{e}} E_{e}}{3} \delta_{N}^{3 / 2}$
Where $N$ is the normal contact force, $\delta_{N}$ is the virtual overlap 258 between the spheres, $R_{e}$ is the effective curvature and $E_{e}$, the 259 equivalent elasticity modulus, is calculated as

$\frac{1}{E_{e}}=\frac{1-v_{1}^{2}}{E_{1}}+\frac{1-v_{2}^{2}}{E_{2}}$

where $v$ is the Poisson ratio of the material. Thus, if we move from the 262 contact between a particle and a metal plate, for which $\left(R_{1} \rightarrow \infty, 263\right.$ $\left.E_{1} \gg E_{2}\right)$ to a contact with two identical spheres $\left(R_{1}=R_{2}, E_{1}=E_{2}\right)$ the 264 stiffness of Eq. (6) diminishes by a factor $2 \sqrt{2}$. For the damping 265 parameter in Eq. (1), no such derivation can be made. However, 266 Pöschel and Schwager [17] proposed following heuristic form for a 267 visco-elastic contact force model:

$N=\frac{4}{3} \sqrt{R_{e}} E_{e}\left(\delta_{N}^{3 / 2}+\frac{1}{2}\left(C_{1}+C_{2}\right) \dot{\delta}_{N} \delta_{N}^{1 / 2}\right)$

where $C_{1,2}$ are damping constants, linked to the material's viscosity 269 [2]. Assuming that the steel plate material reacts purely elastically 271 during collision $\left(C_{1} \approx 0\right)$, this would imply that changing from a 272 particle-hard material interaction to identical particles interaction 273 $\left(E_{1}=E_{2}, C_{1}=C_{2}\right)$, the damping coefficient would be doubled. However, 274 there is no experimental evidence for this. Dintwa [4] showed that a 275 coefficient, approached by:

$\frac{1}{C}=\frac{1}{C_{1}}+\frac{1}{C_{2}}$

yields better agreement with finite element analysis, although an 277 offset value was found compared to FEM. Another drawback is that 279 values become unrealistic when one of the materials has zero 280 damping.

The case of a spherical particle colliding with a cylindrical object 282 (see further), is even more complicated. Unlike the previous case, 283 where the contact surface between the objects is circular, it is now 284 generalized to an elliptical shape. For the elastic normal contact, 285 Eq. (6) is then transformed [10] to

$N=\frac{4}{3} \sqrt{R_{e}} E_{e} \delta_{N}^{3 / 2} F\left(R_{1} / R_{2}\right)$

In this formula, we have

$\frac{1}{R_{1}}=\frac{1}{\infty}+\frac{1}{R_{S}}$ and $\frac{1}{R_{2}}=\frac{1}{R_{S}}+\frac{1}{R_{C}}$.

$R_{1}$ and $R_{2}$ are defined as the principal equivalent radii of curvature 292 of the two indenting bodies $\left(R_{S}\right.$ and $R_{C}$ are the radii of the sphere and 293 the cylinder respectively). Further, $R_{e}$ is defined as

$R_{e}=\sqrt{R_{1} R_{2}}$.

The function $F\left(R_{1} / R_{2}\right)$ is actually a correction which accounts for 297 the eccentricity of the ellipse. Note that if $R_{c} \gg R_{s}$, we have $R_{1} \approx R_{2}$ and 298 $F\left(R_{1} / R_{2}\right) \approx 1$, because the contact area will lose its eccentricity; hence 299 Eq. (10) will again describe the contact between a particle and a plane. 300 Moreover, even if $R_{c} \approx R_{S}$, which is the case for the edges of the vanes, 301 $F\left(R_{1} / R_{2}\right)=0.98$. Hence it is reasonable to use $F=1$ in all cases for the 302 simulations.

In the simulations, the contact stiffness between particle and steel 304 plate $k_{N}$ was set to $2 \mathrm{e} 7 \mathrm{Nm}_{\lambda}^{-3 / 2}+/-3 \mathrm{e} 6 \mathrm{Nm}_{\lambda}^{-3 / 2}$, a value that was 305 obtained by means of an Universal Testing Machine (UTS), while the 306 damping coefficient $c_{N}$ was determined as $140 \mathrm{~m}^{-3 / 2} \mathrm{~s}^{-1}$ using trial and 307 error until the model gives comparable results with experimental 308 rebound tests (e.g. comparing restitution coefficients) [24].

309

The dynamic friction coefficient $\mu$ between fertilizer and disc/vane 310 was obtained by letting the particle slide on a steel plate until a 311 constant particle velocity is reached. By measuring the slope of the 312 


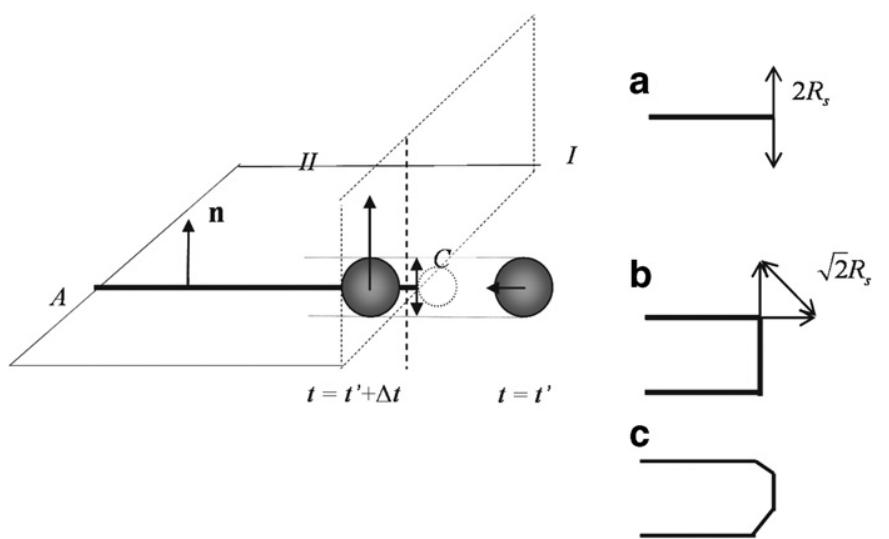

Fig. 6. Particle crossing an edge; right: arrows denote the bad contact area in case of a plane with zero thickness (a), beam (b). (c) depicts a polygonal edge which can further reduce the bad contact area.

plate, the friction coefficient can be determined. In the experiment, an average value of $0.35+/-0.08$ was measured. Since the friction coefficient between fertilizer particles is hard to determine, the same value determined for fertilizer/plate was assumed. ${ }^{2}$

\subsection{Contact detection}

Basically, a particle can have 4 different potential contact partners; namely: another particle, a vane, a vane edge or the disc [24]. The search for possible contacts is done pair wise with the machine parts (vanes, disc, and edges). For particle-particle contact, a more efficient algorithm (Verlet List algorithm [17]) is necessary. Note that all machine parts are geometrical surfaces which are non-closed. Hence, a particle may make contact with an edge of this surface. In some cases, this can lead to unexpected behaviour, as described in the next section.

\subsubsection{Handling particle-edge contacts}

An important issue is that a problem may appear when a particle should hit an edge of a plane $A$. Since all parts are represented as nonclosed hyper-surfaces in the 3D space, they actually have no thickness. As a consequence, when a particle approaches the object from a direction as depicted in Fig. 6, a non-continuous - and possibly very large - overlap will rise as the particle moves from the half-space $I$ to half-space $I I$, defined by the edge of the plane, in the time step $t^{\prime} \rightarrow t^{\prime}+$ $\Delta t$, see Fig. 6 . The particle will be immediately forced to move away from the plane. As the contact force increases with the virtual overlap to the power $3 / 2$, contact forces which are many orders of magnitude larger than the realistically plausible can arise from one time step to the other, leading to an 'explosion' of the particle assembly. Fig. $6 \mathrm{~b}$ illustrates that the problem is not related to the zero thickness, but rather to the presence of a singular edge contact zone, i.e. one with an infinitesimally small local radius of curvature. An area generic solution to the problem was proposed by Tijskens [22] in the framework of particles with arbitrary shape.

To quantify this problem, one can define the probability $P$ of an edge being badly hit by a particle as

$P=P_{\pi} P_{v}$

with $P_{\pi}$ being the probability that a particle is in a space-sector defined by the edge of the plane ( $I$ in Fig. 6 ), and $P_{v}$ the probability the particle has the right approaching velocity. This velocity is dependent on the area (denoted by the double arrows in Fig. 6) which would

\footnotetext{
${ }^{2}$ Particle-particle friction could be higher the wall-particle friction, based on the higher observable smoothness of the vane surface.
}

result in a non-realistic contact force if a particle would cross it. If we 352 assume the particle to be at a distance $d$ from the edge then:

$P_{v} \sim \frac{A}{d^{2}}$

In the case of a single plane (a), we have:

$P_{\pi}=\frac{2 \pi}{4 \pi}$ and $P_{v} \sim \frac{2 L R_{S}}{d^{2}}$

with $L$ the length of the plane edge. This results in:

$P \sim \frac{L R_{S}}{d^{2}}$

In case of a beam (b), we have:

$P_{\pi}=\frac{\pi}{4 \pi}$ and $P_{v} \sim \frac{\sqrt{2} L R_{s}}{d^{2}}$,

hence:

$P \sim \frac{\sqrt{2} L R_{S}}{4 d^{2}}$

yielding a probability of a bad contact with an edge $\sqrt{8}$ times smaller 365 for a beam than for a single plane. Giving this beam a polygonal edge 366 (see Fig. 6c) would further reduce this probability, but one could also 367 use a cylinder which represents the thickness of the vane. Yet, a trade 368 off has to be found in computational efficiency and accuracy. A 369 representation of a vane object for which no false contacts show up is 370 too expensive here.

In this paper, vanes are constructed based on the particle flow 372 direction (see Fig. 7). Single planes with a cylindrical edge are used 373 because the vane thickness is 200 times smaller than the length. As 374 such, the probability for bad contacts is minimal while the computa- 375 tional efficiency is optimal.

3.3. Simulation of the particle flow in the bin

DEM simulations are quite time consuming because at each time 378 step one must find out which particles are in contact and thus 379 subject to contact forces. The time step is determined by the 380 duration of a collision, which decreases with the contact stiffness 381 $k_{N}$, and thus with the equivalent Young's modulus. As a rule of 382 thumb, the number of time steps during a collision should not be 383 less than 20, to ensure proper integration of the contact forces. In 384 order to reduce the computational work, the number of particles is 385 reduced by mimicking the funnel as a tube with the same outlet 386 diameter as the funnel (see Fig. 8). The tube is filled with a number 387 of particles generated randomly and depositing them in the tube 388 under the action of gravity, pretty much as how the funnel is filled in 389 the experiment. A random number generator is used to generate the 390

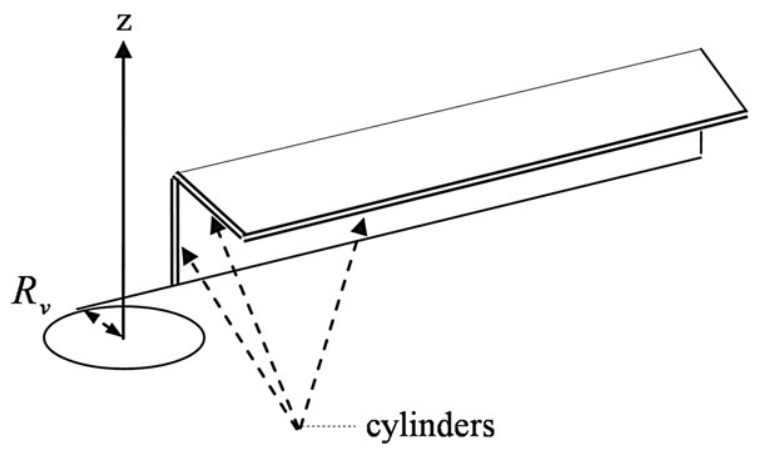

Fig. 7. Handling the vane edges: double lined edges represent a cylinder attached. 

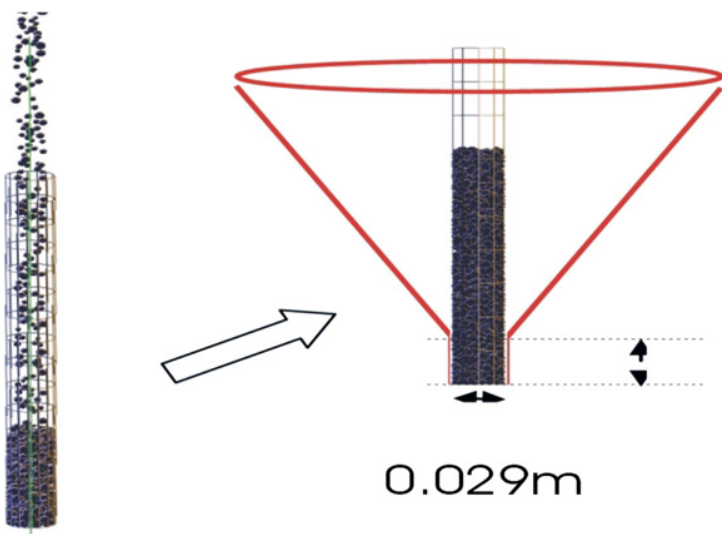

$0.029 \mathrm{~m}$

Fig. 8. Geometry and filling of the tube that mimics the experimental funnel in the simulations. Left: gravitational deposition of particles in the tube, right: relation between the experimental funnel and the simulated tube.

particle radius according to the desired statistical distribution. To ensure that the particles come out of the tube at the desired flow rate, the velocity, $\mathbf{v}$, of the particles in the tube is frozen at a value corresponding to the flow rate:

$\mathbf{v}_{\text {bin }}=-Q_{\text {exp }} / A \rho_{\text {bulk }} \mathbf{e}_{z}$.

Here, $Q_{\exp }$ is the experimental mass flow, $A$ the cross section of the tube, $\rho_{\text {bulk }}$ the fertilizer's bulk density, and $\mathbf{e}_{z}$ the unit vector in the vertical direction. Since high speed camera observations revealed that $\left\|\mathbf{v}_{\text {bin }}\right\|$ from Eq. (19) is underestimated and $A$ is actually smaller (the particles moving out of the bin do not use the whole cross-sectional surface, but move instead faster downwards). By means of a high speed camera, $A$ was proportionally recalculated using the observed $\left\|\mathbf{v}_{\text {bin }}\right\|$. Thus, rather than being accelerated due to the action of gravity, the particles move downwards at constant speed. Also, the wall friction is switched off until just before the particles are going to leave the tube, when gravity and wall friction are switched back on and the particles start to accelerate. The height $\varepsilon$ of the zone where the particles are subjected to gravity and wall friction has no significant influence on the outcome, as long as it is smaller than a few centimetres. Subsequently, the particles fall on the disk, are caught by the vane, and are thrown into the collector bins. Once it is known in which bin the particle will end up, it is useless to the simulation. However, rather than taking it out of the simulation, the particle is recycled back into the tube (see Fig. 9) where, once they reach the region indicated by $\varepsilon$, their velocity is frozen to the value of its
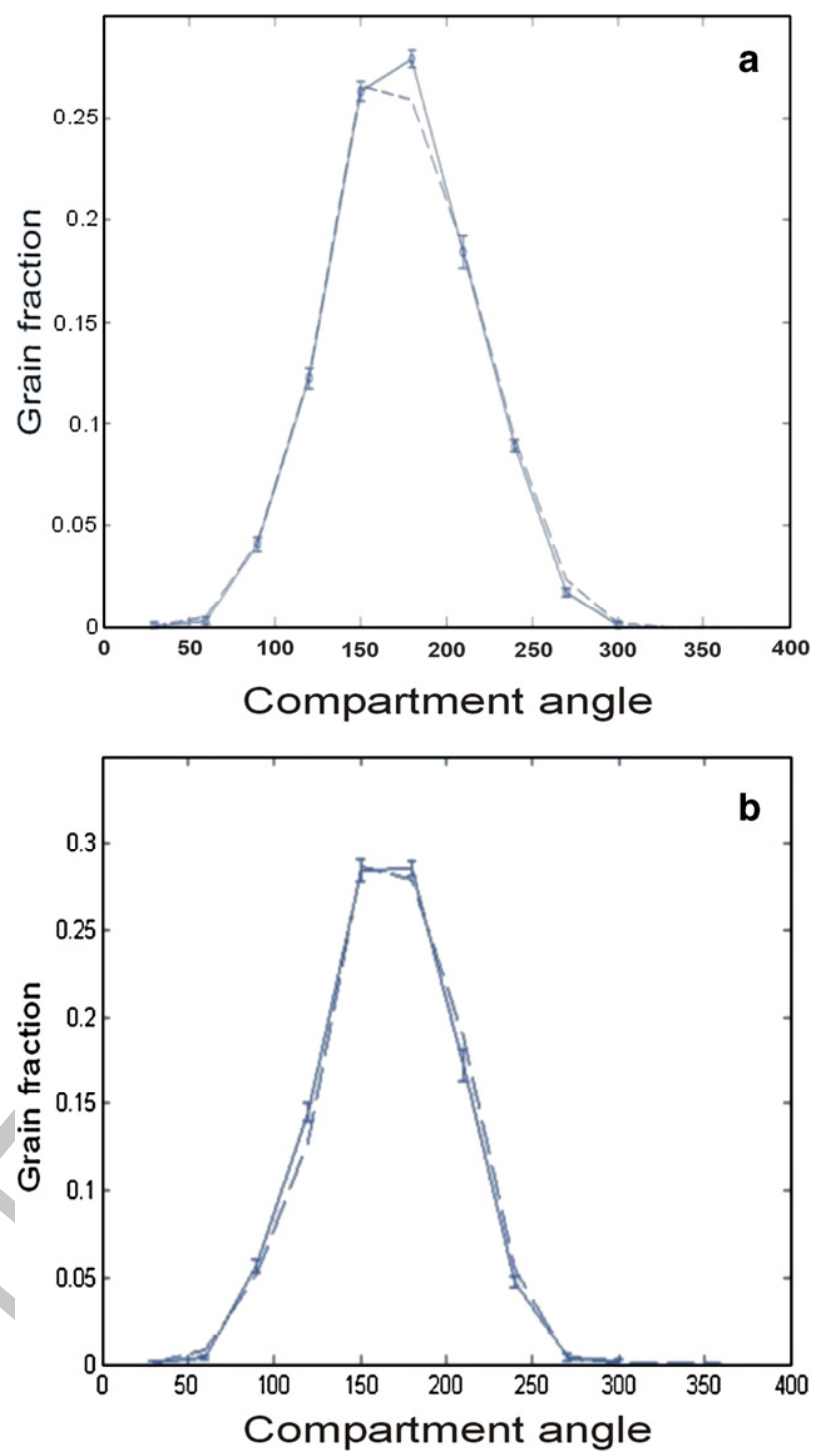

Fig. 10. (a) Cylindrical plots of experiment (solid lines) and DEM simulation (dashed line) for disc type A, $\Omega_{d}=300 \mathrm{rpm}$; (b) Cylindrical plots of experiment (solid lines), and DEM simulation (dashed line) for disc type A, $\Omega_{d}=400 \mathrm{rpm}$. Error bars are shown.

neighbouring particles. In this way the minimum number of particles 417 that is needed, is determined by the requirement that the tube should 418 never run empty.

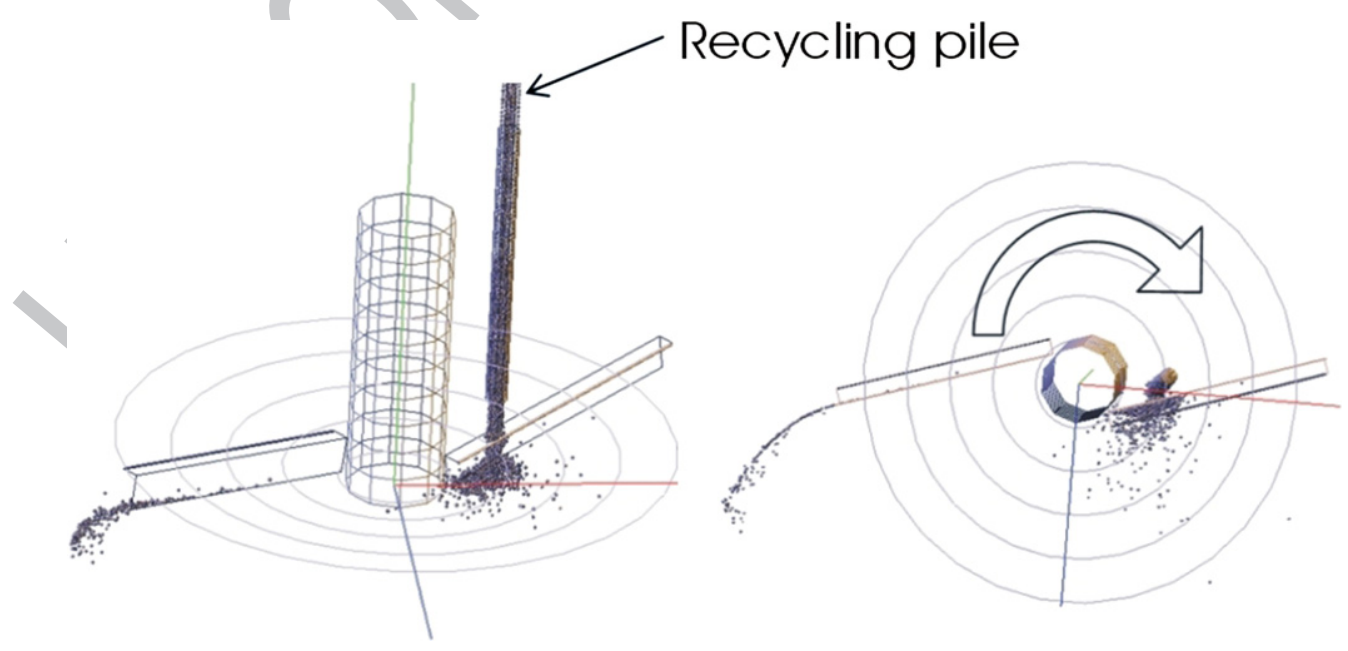

Fig. 9. Snapshot of a DEM simulation (for Type B disc): side view and top view. 
The rotating disk is implemented as a conical disk while the Lshaped vanes are implemented as two planes of zero thickness. The contact problem discussed in Section 3.2.1 of the contact with the singular edges is solved by 'gluing' cylinders along the edges as depicted in Fig. 7. Only three cylinders need to be added, the remaining edges are either hidden by the connection of the vane to the cone, or have zero probability of catching a particle in the given confirmation and dynamics. A snapshot of a simulation is shown in Fig. 9.

In the experiments, a natural variance of the particle positions can be expected since each bin filling is different. The measured bulk density in the funnel was $1040 \mathrm{~kg} / \mathrm{m}^{3}$ versus $1050 \mathrm{~kg} / \mathrm{m}^{3}$ in the simulated tube. By means of Eq. (19) one obtains $A=0.00035 \mathrm{~m}^{2}$ with $Q_{\exp }=0.11 \mathrm{~kg} / \mathrm{s}$ and $\left\|\mathbf{v}_{\text {bin }}\right\|=0.3 \mathrm{~m} / \mathrm{s}$. In the simulation, a minimum of 7000 particles is needed, having a theoretical equivalent of approximately $107 \mathrm{~g}$ fertilizer. Due to the recycling of particles, the actual mass of fertilizer that is spread in the simulation is $Q_{e} T_{\text {sim }}$, with $T_{\text {sim }}$ the simulated time span.

\section{Results and discussion}

\subsection{Output variables}

In order to have a good comparison between simulation and experiment, 4 different rotation speeds of the disc were chosen: $300 \mathrm{rpm}, 400 \mathrm{rpm}, 500 \mathrm{rpm}$ and $650 \mathrm{rpm}$. At each speed, the test was repeated 5 times to average out possible variations due to the unknown position of the vanes at $t=0$.

Especially with high disc speeds, particles may suffer high breakage and some are degraded to a coarse dust, which easily sticks to some parts of the collectors. Even when finer remains are removed from the metal parts and added to the respective collector, a loss of a small fraction can be attributed to impacts with the vane edges (bouncing) or the metal divisions between the collectors. In the simulation however, this will not appear since the particles' positions are simply written down when they reach a distance of $0.50 \mathrm{~m}$ from the centre. To account for this, it is better to compare particle fractions in each compartment rather then the collected mass.

Table 1

Summarize of the characteristics of the CSP measurements and comparison with DEM

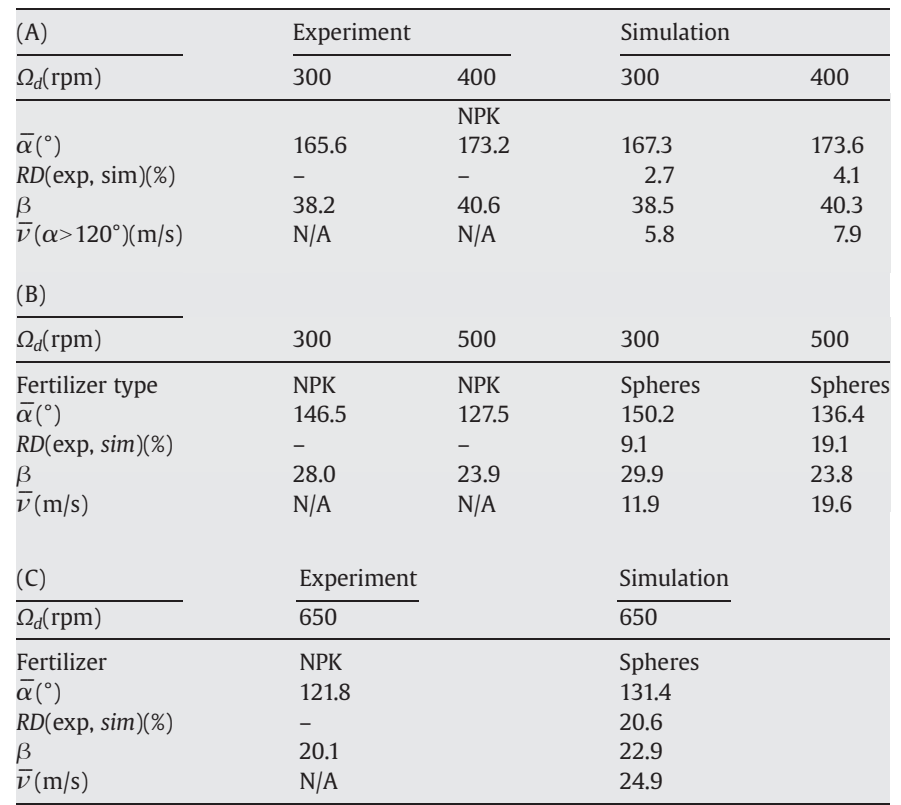

(A) Disc type A with $\Omega_{d}=300 \mathrm{rpm}, 400 \mathrm{rpm}$. (B), (C) for disc type B with $\Omega_{d}=300 \mathrm{rpm}$, $500 \mathrm{rpm}$ and $650 \mathrm{rpm}$ respectively.
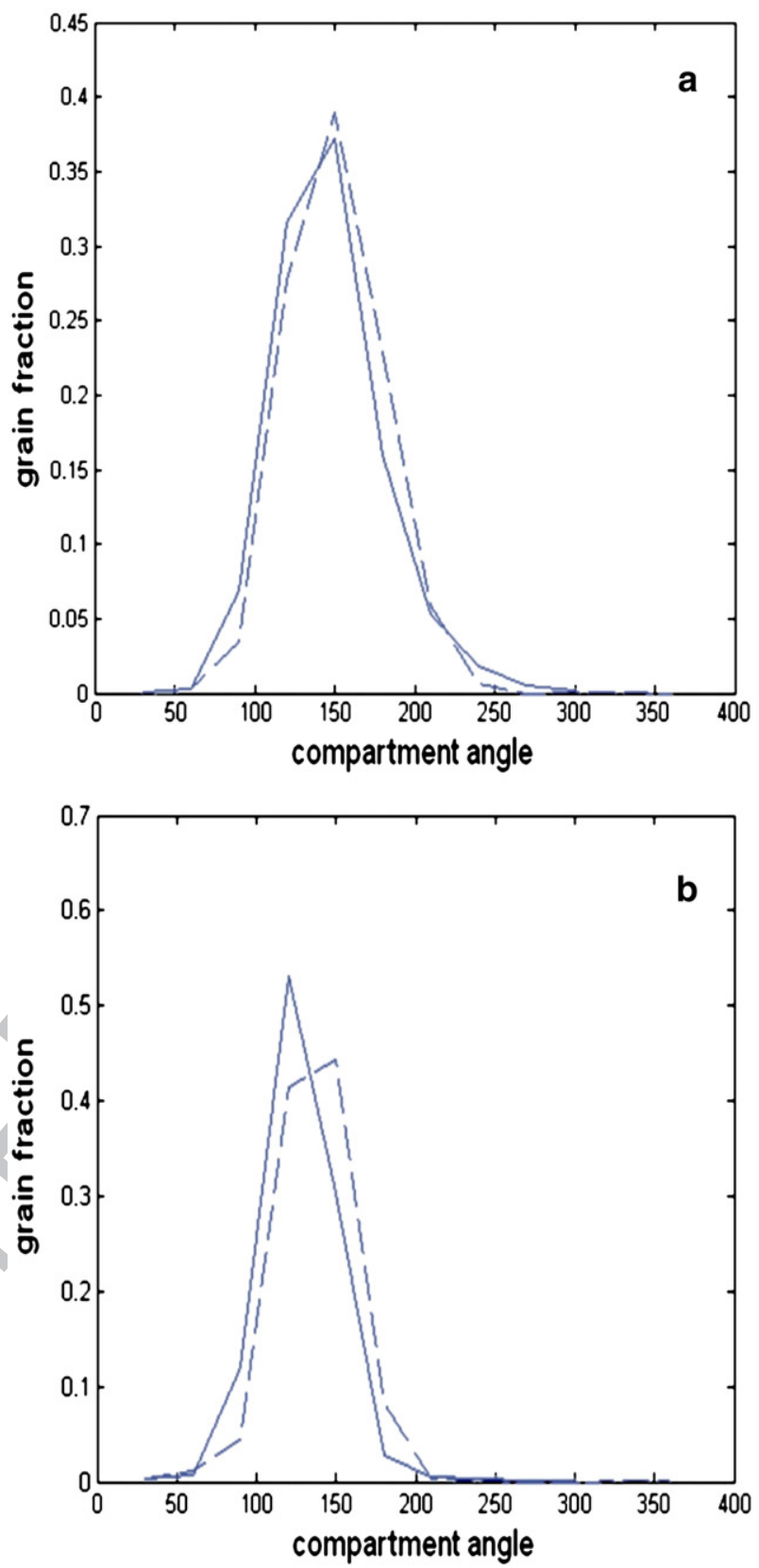

Fig. 11. Cylindrical plots of experiment (solid lines) and DEM simulation (dashed line), for (a) $\Omega_{d}=300 \mathrm{rpm}$ and (b) $\Omega_{d}=500 \mathrm{rpm}$ for disc type B. Error bars are omitted.

To summarize an experiment, the average distribution angle for 454 the CSP is defined as:

$\bar{\alpha}=\sum_{i} f_{i} \alpha_{i}$

with $\alpha_{i}$ the angle of each $i$-th compartment and $f_{i}=\frac{M_{i}}{M_{\text {tht }}}$ the fraction of 457 particles. One may also introduce a measure for the width of the 458 pattern, $\beta$ :

$\beta=\sum_{i} f_{i}\left|\alpha_{i}-\bar{\alpha}\right|$

After manipulation of the data of the model output, a comparison 462 can be made with the data from experiments. Olieslagers [14] 463 proposed a deviation function (RD) for comparing simulations and 464 experiments, using the sum of absolute differences which can be used 465 for both CSP and TSP:

$\mathrm{RD}(\exp , \operatorname{sim})=\frac{\sum_{i}\left(\left|M_{i, \exp }-M_{i, \text { sim }}\right|\right)}{M_{\text {tot,exp }}+M_{\text {tot }, \text { sim }}} 100$. 


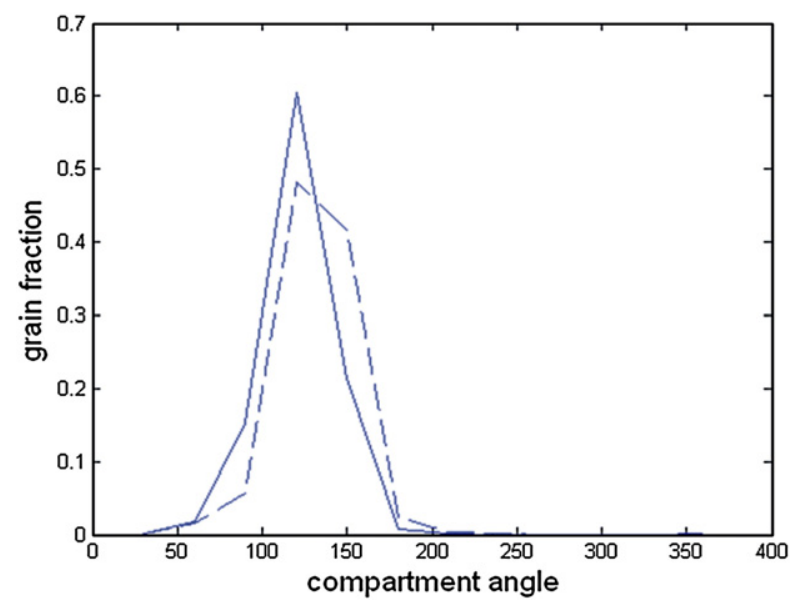

Fig. 12. Cylindrical plots of experiment (solid lines) and DEM simulation (dashed line), for $\Omega_{d}=650 \mathrm{rpm}$ for disc type. Error bars are omitted.

\subsubsection{Cylindrical measurements}

Generally, the results of simulations and experiments are best viewed in a plot of the grain fraction as a function of the compartment degree. The distribution along the compartments is bell-shaped; in the first compartments, there are less grains because these segments are almost at the same angle as the orifice position and most of the particles have not yet left the disc. Conversely, for the furthest compartments, almost all the particles have left the disc, hence the lower amount of grains collecting there. Fig. 10a and b give these distributions for a disc type A with a rotational speed of $300 \mathrm{rpm}$ and $400 \mathrm{rpm}$ respectively. The dashed lines in the figures are for simulations while solid lines are for experimental data. The width of the error bars is $+\mid-2 \sigma$, where $\sigma$ is calculated from 5 data points.

Fig. 10 clearly shows a very good agreement between simulation and experiment, with most points lying in the range of the error bars, but with a small deviation in the $200^{\circ}$ compartment. For the rotational speed of $400 \mathrm{rpm}$, the simulation results again are in good agreement, although a small offset to the right side is established.

From Table $1(A)$, showing all the characteristics of the simulated and experimental patterns, one concludes that the simulation output is very close to the experimental data of NPK measurements; values for $R D$ (exp, sim) are less that $5 \%$.

Measurements with the field spreader disc (type B) were done for disc rotational speeds of $300 \mathrm{rpm}, 500 \mathrm{rpm}$, and $650 \mathrm{rpm}$. The results are shown in from Figs. 11 and 12 respectively. One notices that the curves gradually shift to the left (e.g. lower $\alpha$ values), because the particles leave the disc earlier; and also get narrower (e.g. $\beta$ decreases). The error bars, which are comparable with previous experiments, are omitted to have a better view.

The simulated grain distributions are also shown in Figs. 11 and 12 by dashed lines. A comparison between simulation and experimental curves shows that, for a disc speed of $300 \mathrm{rpm}$, the DEM simulations approximate the experimental curve quite well (deviations are $~ 10 \%$ ), but for the $500 \mathrm{rpm}$ case, the deviations become substantial (19\%) while for $650 \mathrm{rpm}$ they are even higher (21\%). Moreover, all simulated curves are offset to the right (higher $\alpha$ values), meaning the particles leave the disc later, and the greater the disc speed the more this is pronounced. The results of the experiments are further summarized in Table 1 (B, C).

\subsubsection{Rectangular measurements}

The measurements of the total spread pattern, were done on an area of $25 \mathrm{~m}^{2}$ covered with a plastic foil. Because of the spatial limitations on the measuring hall, the test for this experiment was only performed by a lawn spreader disc (type A). The main advantage of experiments with this type is that their range is small (not more than $3 \mathrm{~m}$ working width).
Hence, the TSP can be measured in a relatively small space. Furthermore, 514 the particles have a relatively low velocity, which makes them less 515 susceptible to deviations induced by aerodynamic forces. Measurements 516 with the conical disc were technically not feasible.

Generally, there are 11 experiments and $15 \times 11=165$ measuring 518 points required to cover the whole area of the spread pattern. 519 However, one can also skip lines in order to reduce this number but 520 still have enough control points. In total, $3-5$ lines were considered at 521 a distance $^{3}$ from $\mathbf{Y}=0 \mathrm{~m},-0.5 \mathrm{~m},-1 \mathrm{~m},-1.5 \mathrm{~m}$, and $-2 \mathrm{~m}$ to the disc 522 centre, depending on the disc rotational speed. To minimize the 523 interference from particles in neighboring baskets, a soft layer of 524 material was placed against the walls of the baskets. In this manner, 525 particles cannot jump out of neighboring baskets. Each single 526 experiment was repeated 4 times for each longitudinal distance in 527 order to have a good repeatability. The disc rotation is clockwise. $\quad 528$

In Fig. 13, a contour plot of the total area covered by the measured 529 and simulated pattern is given for a disc rotational speed of $400 \mathrm{rpm} .530$ The missing lines $\mathbf{Y}=-0.25 \mathrm{~m}, \mathbf{Y}=-0.75 \mathrm{~m}, \mathbf{Y}=-1.25 \mathrm{~m}$ and $\mathbf{Y}=-1.75 \mathrm{~m} 531$ were obtained by interpolating between the measured lines. The total 532 area covered by the measurements is approximately $9 \mathrm{~m}^{2}$ which 533 resulted in $94 \%$ of the total particle mass used in the experiment. The 534 remaining fraction missing is due to particles that land beyond the 535 basket area and the interpolation deviations between the lines. $\quad 536$

The pattern has a typical bended shape around the disc, with a high 537 concentration zone in the front of the disc. Besides this there was also an 538 increase of density on the right side of the disc. This small "peak" 539 corresponds to the particles bouncing from contact with the vane edges 540 and spill-over. The effect is probably somewhat exaggerated with respect 541 to the type B discs, because the latter mostly have horizontal flaps which 542 actually limit this spill, whereas the lawn spreader disc has not. 543

In Fig. 14, the particle distributions of the lines $\mathbf{Y}=0 \mathrm{~m},-0.5 \mathrm{~m}, 544$ $-1 \mathrm{~m},-1.5 \mathrm{~m}$, and $-2 \mathrm{~m}$ are plotted against the transverse distance $\left(\mathbf{X}_{545}\right.$ direction) for a disc speed of $400 \mathrm{rpm}$. The error bars representing the 546 $[-2 \sigma 2 \sigma]$ range are given only for Fig. 14 and b for clarity. For the 547 validation of the model, the output files from the same simulations as 548 in Section 4.1.1 were used as input for the ballistic model. For the 549 ballistic flight, a drag coefficient of $C_{p}=0.44$ is used [14]. Subsequently, 550 a post processing program calculates the distributions on the ground, 551 and calculates the respective transverse distributions. The results of 552 the DEM simulations are denoted by the dashed lines.

553

From Fig. 14a, b and c, giving the patterns for $\mathbf{Y}=0 \mathrm{~m}, \mathbf{Y}=-0.5 \mathrm{~m}$ and 554 $\mathbf{Y}=-1 \mathrm{~m}$, we conclude that the DEM simulations predict the pattern 555 qualitatively well (e.g. shape of the curve), but some overestimations of 556 the particle collected masses are observed at all distances. In the overall 557 view, Fig. 13 shows that the experimental pattern in radial direction, e.g. 558 distance from the disc centre, is wider than the simulated. In other 559 words, the simulated pattern is generally smaller in surface and more 560 concentrated in the radial direction than the experimental one.

In Table 2, the results are further summarized, giving the total 562 particle mass per line and the deviation of the DEM simulation. The 563 relative average deviation (RD) per line $\mathbf{Y}$, given by:

564

$\mathrm{RD}_{Y}(\exp , \operatorname{sim})=\frac{\sum_{x}\left(\left|M_{X Y, \exp }-M_{X Y, \operatorname{sim}}\right|\right)}{M_{Y, \exp }+M_{Y, \text { sim }}} 100$

varies around $25 \%$. The fact that these deviations are much larger for 566 the similar cylindrical experiment, is due to a number of reasons: $\quad 567$

1. The low angular resolution of the baskets $\left(30^{\circ}\right)$ averages deviations 568 too much.

2. A fraction (2\%) of the mass is not captured because of bouncing in 570 the Carrousel.

3. The cylindrical measurements give no information about the 572 velocities. 

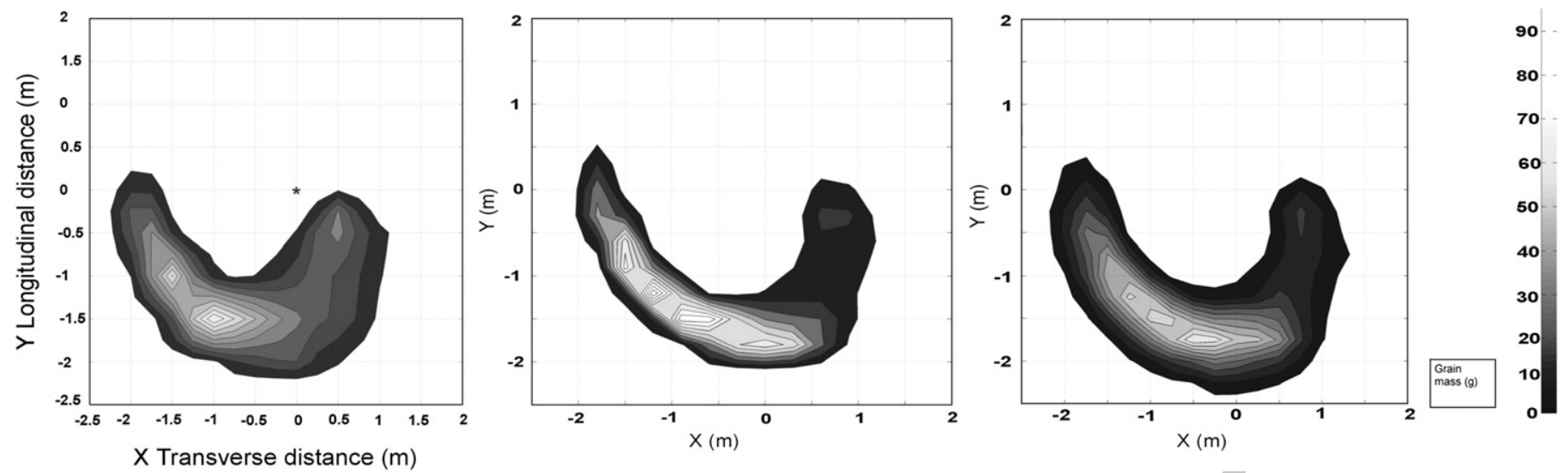

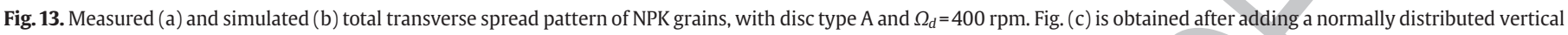
random component to the particle disc edge velocity (see Section 4.2$)$. The location of the disc is indicated by $(*)$.

\section{An additional deviation is made in the ballistic flight calculation} (aerodynamic properties).

To exclude the uncertainty about the influence of the measurement errors for $D_{\text {bin }}, R_{\mathrm{bin}}, h_{\mathrm{bin}}, \Omega_{\mathrm{d}}$, and $L_{\mathrm{vane}}$, additional simulations were run with a variation of $2 \%$ of these dimensions. This however did not have any significant improvement on the output.

Table 2 also shows that the sum of the predicted mass over the transverse direction $\left(M_{Y}\right)$ is in good agreement with the experiments, which confirms that the qualitative prediction of the model is good.

Additional measurements were carried out for a disc speed of $300 \mathrm{rpm}$. In Fig. 15 the fertilizer mass distribution (solid lines) is only given at lines $\mathbf{Y}=-0.5 \mathrm{~m}, \mathbf{Y}=-1 \mathrm{~m}$ and $\mathbf{Y}=-1.5 \mathrm{~m}$, because the range of the pattern is smaller. For the comparison, the DEM simulations are indicated by the dashed lines. Fig. 15 shows that compared to the $300 \mathrm{rpm}$ measurements the agreement is much better here, with a fairly good overlap, except for the collector line most distanced from the disc centre, where a significant underestimation is observed. Table $2 \mathrm{~B}$ reads that the deviations are generally lower than for the $400 \mathrm{rpm}$ experiment, although the same significant deviation is observed for the most distanced line.

\subsection{Discussion of the results}

Referring to single particle models and experiments [9,15,24], a higher $\alpha$ value of a single particle trajectory can be explained by (1) an initial position of the particle being nearer to the disc center, (2) a smaller initial outward radial particle velocity on the disc, or (3) a higher opposing force on the particle moving on the disc, resulting in a particle velocity and hence a later exit off the disc. Although no multi particle experimental tests were conducted, explanations (1) and (2) were not considered, based on the fact that the average initial positions and velocities of the particles leaving the bin are quite accurately specified in the experiment. Also, additional test simulations considering the possible variations of the average positions and velocities ${ }^{4}$ of maximum $2 \%$ did not give any satisfying improvement. Explanation (3) could be a result of either a measurement error of the friction coefficient in the tangential contact model, or a measurement error of the damping in the normal force model. A model parameter study using a flat disc rotating at $500 \mathrm{rpm}$, depicted in Fig. 16a, shows that the DEM simulations show a high sensitivity to friction coefficients in the CSP plots (e.g. the curves shift to lower average outlet angles when using lower friction coefficients). On that basis,

\footnotetext{
${ }^{4}$ The initial positions and velocities on the disc of the individual particles are not known in the experiment. Their average values when leaving the bin however are known through the accurate position of the bin and the velocity monitoring of the particles with a high speed camera.
}

one could hypothesize that the deviations between simulations and 614 experiments are due to an overestimation of the friction coefficients. ${ }^{5} 615$

This brings us to the idea to adapt the model by extending it with a 616 linear velocity dependent friction coefficient:

$\mu^{*}=\mu+a v$

where $v$ is the tangential wall-particle velocity. To avoid spurious 618 simulation results, only the wall-particle friction coefficient was taken 620 to be velocity dependent. The reason for this is that particles can 621 approach each other with high relative velocity, leading to possible 622 negative friction coefficients. This is especially the case when recycling 623 the particles into the bin. The friction model as defined by Eq. (3) 624 would therefore be too simple.

To find the optimal value of $a$ in Eq. (24), a Matlab® Isqnonlin 626 algorithm applied to the differences of the average outlet angles 627 between simulation and experiments for disc type B was used. In 628 Fig. 17 the outlet angles are given as a function of the value of $a$ for 3629 rotational speeds. The optimal values are also indicated. A single 630 optimal value of $a=0.02 \mathrm{~s} / \mathrm{m}$ was found to lower all deviations 631 between simulations and experiments to a maximum value of 5\%. 632

Since various researchers $[11,8,14]$ have proposed velocity depen- 633 dent friction coefficients, this strengthens the idea that the friction 634 coefficient is probably overestimated in the measuring methods and 635 should probably be regarded as a speed dependent parameter. From 636 another perspective, the cylindrical measurements might be an 637 interesting (and cheap) tool to investigate (especially) the tangential 638 contact force models and parameters for their speed dependence. $\quad 639$

The underlying mechanisms explaining the difference in the 640 transverse spread patterns (TSP) seem to be more complicated than 641 in the cylindrical experiments. One should remark that, regarding the 642 400 rpm experiments, a similar effect was observed by Reumers et al. 643 (2003) [18] who conducted experiments in a spreader hall and tried to 644 predict the spread patterns by means of a ballistic model and 645 measurements of the particle outlet velocities at the edge of the disc. 646 Reumers suggested that the deviations are most likely due to a slight 647 measurement error on the vertical (Z-direction) start position/velocity 648 at the edge of the disc, giving significantly more spread on the ground 649 positions. To quantify this, assume a projectile with initial velocity $\mathbf{v} 650$ $\left(v_{0 r}, v_{0 z}\right)$, where $v_{r}$ and $v_{z}$ are the horizontal (along the disc surface) and 651 vertical velocity components (perpendicular to the disc) respectively. 652 Assume further the particle leaves at position $\mathbf{r}\left(r_{0}, z_{0}\right)=(0,0)$.The 653

\footnotetext{
5 The deviations are not due to growing numerical errors, as single particle DEM simulations agree well with analytical models [15] for a broad range of disc rotational speeds.
} 
P. Van Liedekerke et al. / Powder Technology xxx (2008) xxx-xxx
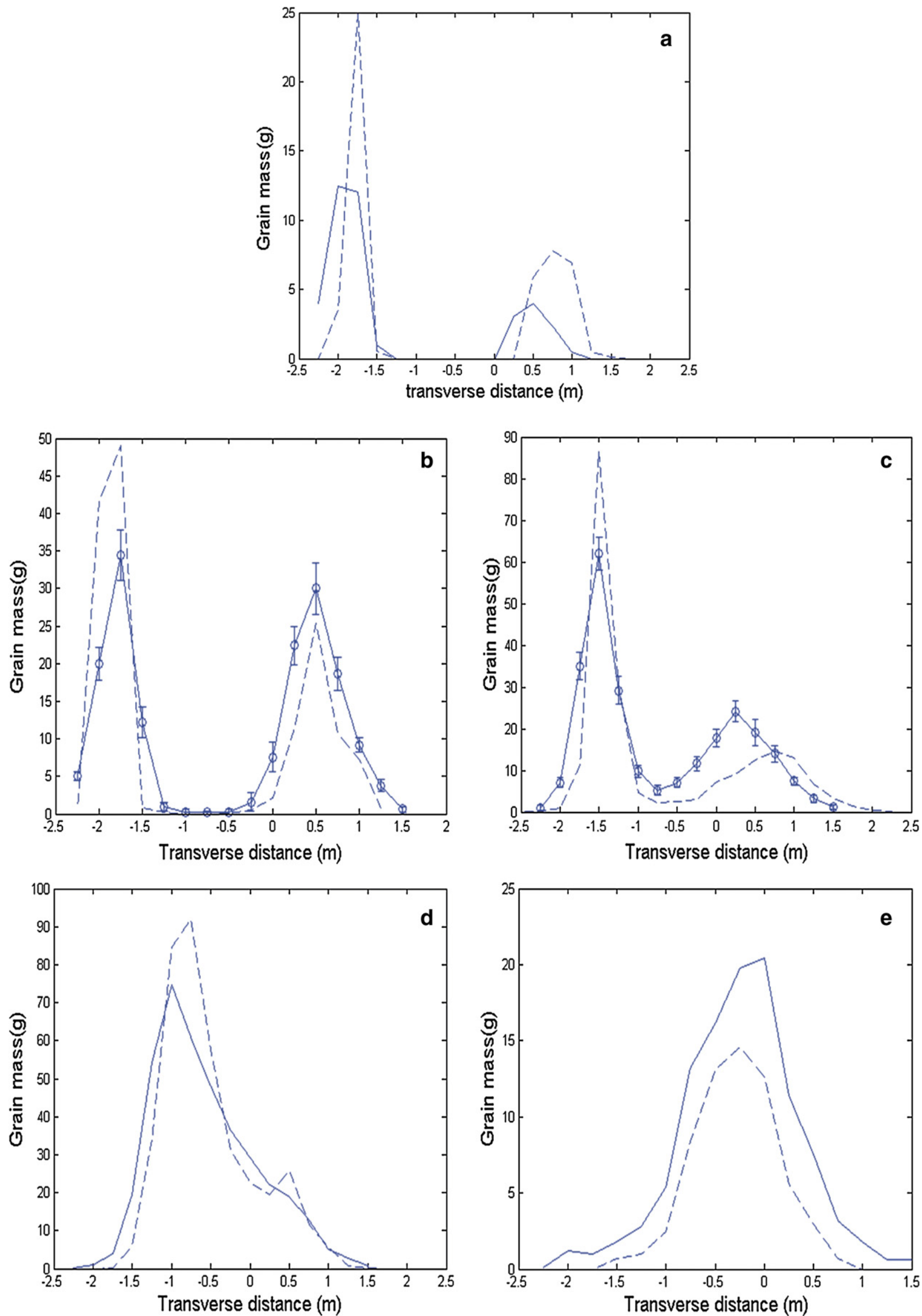

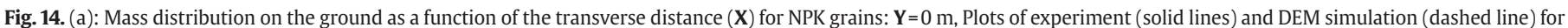

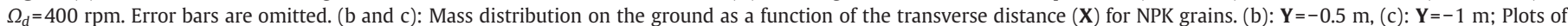

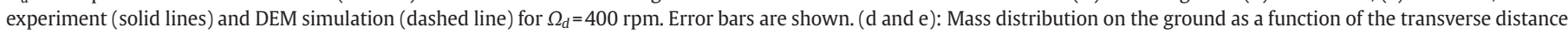
(X) for NPK grains (d): $\mathbf{Y}=-1.5 \mathrm{~m}$, (e): $\mathbf{Y}=-2 \mathrm{~m}$. Plots of experiment (solid lines) and DEM simulation (dashed line) for $\Omega_{d}=400 \mathrm{rpm}$. Error bars are omitted. 
Table

Summarize of the TSP experiment for NPK, (A) with disc speed $400 \mathrm{rpm}$, (B) with disc speed $300 \mathrm{rpm}$

\begin{tabular}{lllll}
$\mathrm{t} 2.2$ & & $\begin{array}{l}\text { Total grain mass } M_{y}, \text { exp. } \\
\mathrm{t} 2.3\end{array}$ & $\begin{array}{l}\text { Total grain mass } M_{y}, \text { sim. } \\
(\mathrm{kg})\end{array}$ & $\begin{array}{l}R D(\mathrm{exp}, \mathrm{sim}) \\
(\%)\end{array}$ \\
\cline { 2 - 5 } $\mathrm{t} 2.4$ & $(\mathrm{~A})$ & & 0.59 & 35.2 \\
$\mathrm{t} 2.5$ & $\mathbf{Y}=-0 \mathrm{~m}$ & 0.40 & 0.171 & 25.5 \\
$\mathrm{t} 2.6$ & $\mathbf{Y}=-0.5 \mathrm{~m}$ & 0.163 & 0.236 & 28.4 \\
$\mathrm{t} 2.7$ & $\mathbf{Y}=-1 \mathrm{~m}$ & 0.251 & 0.417 & 13.3 \\
$\mathrm{t} 2.8$ & $\mathbf{Y}=-1.5 \mathrm{~m}$ & 0.390 & 0.64 & 26.8 \\
$\mathrm{t} 2.9$ & $\mathbf{Y}=-2 \mathrm{~m}$ & 0.107 & & \\
$\mathrm{t} 2.10$ & & & & 12.1 \\
$\mathrm{t} 2.11$ & $(B)$ & & 0.233 & 13.3 \\
$\mathrm{t} 2.12$ & $\mathbf{Y}=-0.5 \mathrm{~m}$ & 0.264 & 0.455 & 38.6 \\
$\mathrm{t} 2.13$ & $\mathbf{Y}=-1 \mathrm{~m}$ & 0.497 & 0.035 & \\
$\mathrm{t} 2.14$ & $\mathbf{Y}=-1.5 \mathrm{~m}$ & 0.066 & &
\end{tabular}

$\mathbf{M}_{r}=-\mu_{r}\|\mathbf{N}\| \frac{\omega_{T}}{\left\|\omega_{T}\right\|}$

\footnotetext{
${ }^{6}$ Higher friction also slows down the particles, which is difficult rhyme with was concluded previously.
}

where $\mu_{r}$ is the rolling friction coefficient, and $\boldsymbol{\omega}_{T}=\boldsymbol{\omega}_{\wedge}-\mathbf{n}\left(\boldsymbol{\omega}_{\wedge} \cdot \mathbf{n}\right)$ is the 691 angular velocity of the particle contributing to rolling (i.e. the total 692 angular velocity lowered by the twisting motion between particle and 693 contact surface). The particle flow affected by introducing the rolling 694 friction coefficient is depicted in Fig. 16c. The simulation results are 695 given for $\mu_{r}=0$ (no rolling friction), $\mu_{r}=0.0001$ and $\mu_{r}=0.001$. One 696 clearly observes that a higher rolling friction increases the outlet 697 angles of the particles (thus slowing them down), although the effect 698 is limited because at a certain point the rotational motion of the 699 particles is frustrated and a pure sliding regime is adopted $\left(\mu_{r}>0.001\right) .700$ Another important observation is that the variation of the particle 701
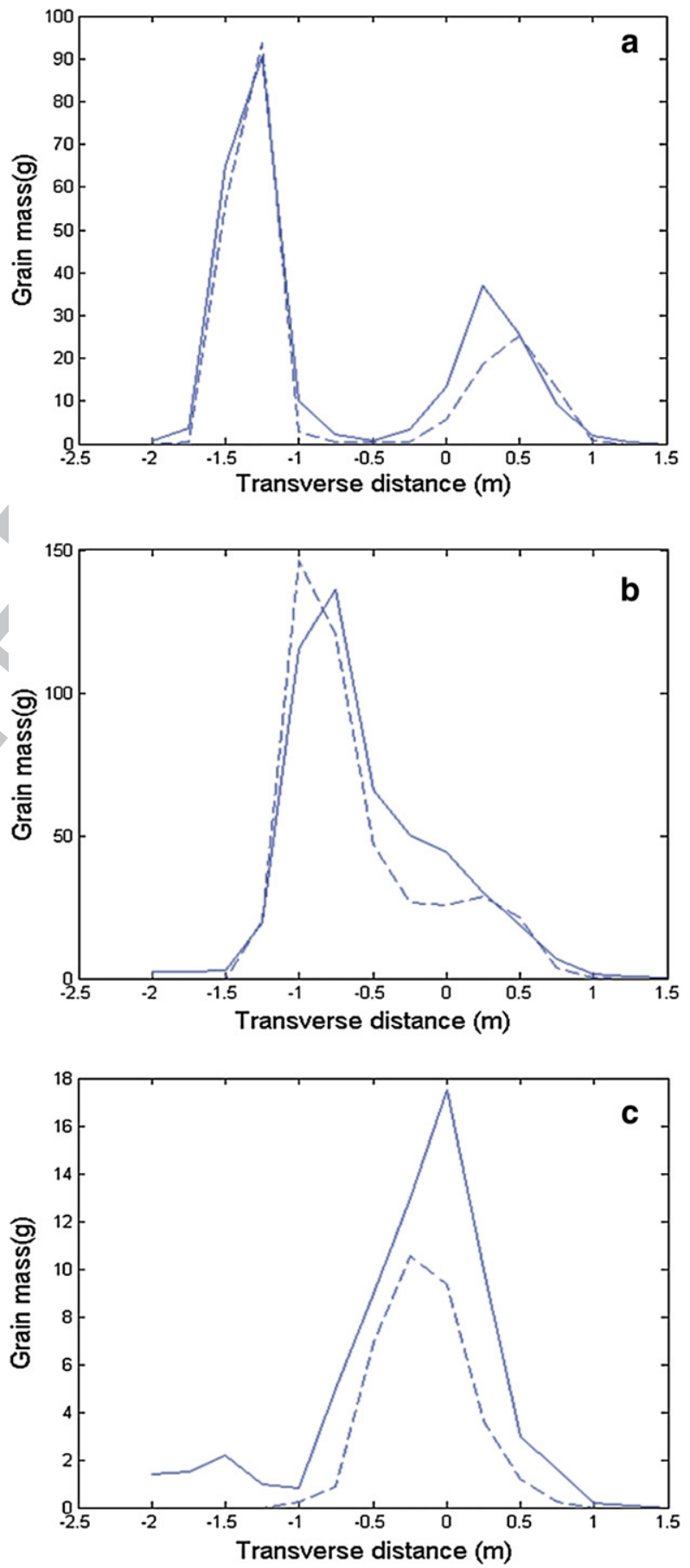

Fig. 15. Mass distribution on the ground as a function of the transverse distance $(\mathbf{X})$ for NPK. (a): $\mathbf{Y}=-0.5 \mathrm{~m},(\mathrm{~b}): \mathbf{Y}=-1 \mathrm{~m},(\mathrm{c}): \mathbf{Y}=-1.5 \mathrm{~m}$, Plots of experiment (solid lines) and DEM simulation (dashed line) for $\Omega_{d}=300 \mathrm{rpm}$. Error bars are omitted. 

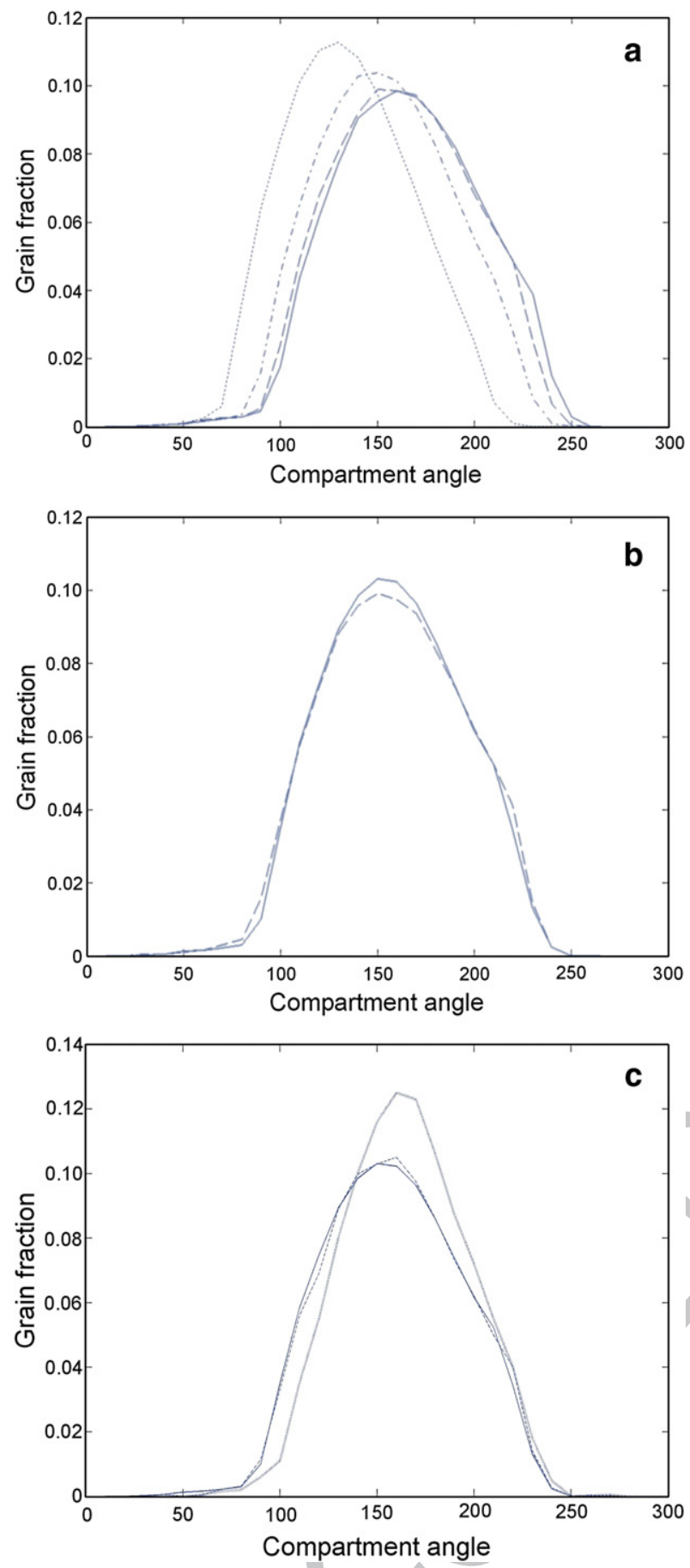

Fig. 16. Simulated cylindrical pattern plots of DEM simulation ( $0.6 \mathrm{~m}$ diameter flat disc rotating at $500 \mathrm{rpm}$ ), showing (a) the influence of friction coefficients using values: 0.1 (dotted line) 0.3 (dash-dotted) 0.5 (dashed) 0.7 solid), (b) the influence of the particle restitution coefficients using values 0.2 (solid line) and 0.8 (dashed line), and (c) the influence of the rolling friction coefficient using values 0.0 (solid line), 0.0001 (dashed line), and 0.001 (dotted line)

velocities and positions at the disc edge is lowered (e.g. CSP width gets smaller). Hence, the rolling friction cannot account for the deviations in the TSP.

In Conclusion, it remains unclear where such an increase in dispersion of the particle speed may originate from. The model parameters, describing the contact force model may have inaccurate values, or the contact model itself may be insufficient. Perhaps, external factors such as small vibrations of the plane of the spinning disc at higher disc speeds (especially when recalling the disc is made 710 of PVC), or the interaction of the particles with the complex airflow 711 around the disc could be responsible. Additional experiments would 712 be recommended in further research.

\section{Conclusions}

In this paper, DEM simulations of a fertilizer particle flow on a 715 spinning disc were compared with experiments. The experiments 716 were conducted in two different ways. The first one is a measurement 717 of the particle distribution around the disc edge, yielding the 718 cylindrical spread pattern (CSP). This is achieved by use of a cylindrical 719 collector tray with segmental baskets each having an angle of $30^{\circ}$. The 720 second method is the measurement of the total spread pattern on the 721 ground. Here, a line of 15 baskets was constructed and positioned at 722 different distances from the disc centre to cover the area of the whole 723 fertilizer mass on the ground.

Overall, it was found that the DEM simulations perform well when 725 the comparison with the experiments is made qualitatively. More 726 specifically, the cylindrical measurements revealed that the prediction 727 of the spread pattern is very good (deviations are below $5 \%$ ) when the 728 particle outlet velocities are low (e.g. $<10 \mathrm{~m} / \mathrm{s}$ ). For higher disc 729 rotational speeds, the deviations grow and the simulations lose their 730 quantitative predictive value, mainly due to an offset to higher outlet 731 angles of the simulated pattern. As a consequence, one could posit that 732 the opposing forces on the particle flow, either the frictional contact or 733 the normal damping, are overestimated. A small sensitivity study 734 revealed that an overestimation of friction coefficients can be 735 responsible for this behavior. From another perspective, the cylind- 736 rical measurements might be an interesting (and cheap) tool to 737 investigate (especially) the tangential contact force models and 738 parameters for their speed dependence.

For the experiments of the transverse spread patterns, a similar 740 conclusion could be drawn, and even though the disc speeds are 741 relatively low, the deviations are clearly magnified compared to the 742 cylindrical measurements. These deviations are mainly due to an over 743 concentration of fertilizer mass in the radial direction compared to the 744 experiments (especially at higher disc speeds). A similar effect was 745 observed by Reumers et al. (2003) [18] who conducted experiments in 746 a spreader hall and tried to predict the spread patterns by means of a 747 ballistic model and measurements of the particle outlet velocities at 748 the edge of the disc. Likely, one could link these deviations to the 749 conclusions made by Reumers. It is then plausible to assume that they 750

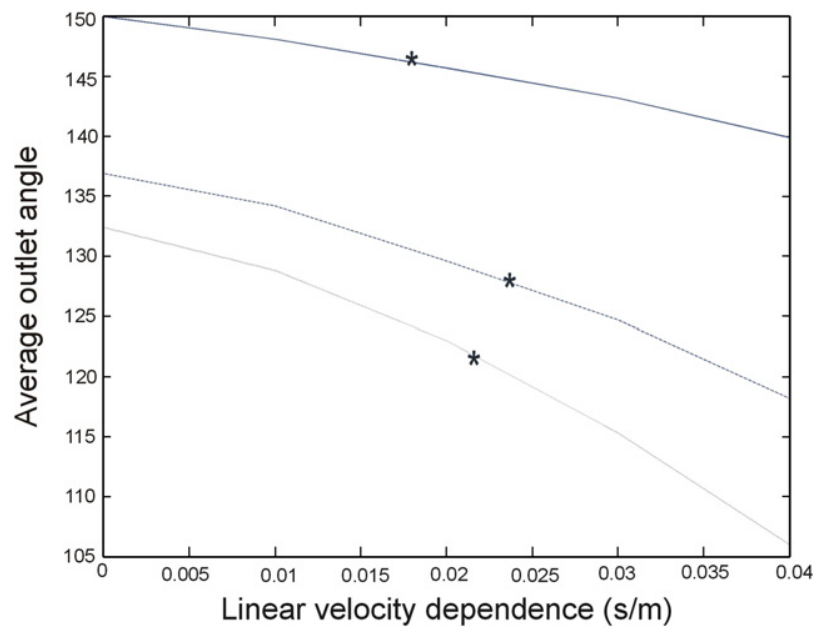

Fig. 17. Simulated outlet angles as a function of the linear velocity dependent friction model parameter $a$ in Eq. (24) for disc type B with $\Omega_{d}=300 \mathrm{rpm}$ (solid line), $\Omega_{d}=500 \mathrm{rpm}$ (dashed line), $\Omega_{d}=650 \mathrm{rpm}$ (dotted line). The optimal values for reducing the deviations with the relative experiments are indicated with a $\left(^{*}\right)$ 
are due to miscalculation of the velocities - with emphasize on the vertical components - at the disc edge.

Since a model parameter study did not give any sense for improvement, one could consider additional experiments under well specified conditions to investigate the influence of vibrations and airflow. Other, more sophisticated, contact force models could also give a new direction as well.

\section{Notation}

\section{Regular symbols}

A (Cross) section area $\left[\mathrm{m}^{2}\right]$

c damping coefficient in Kono-Kuwabara model $\left[\mathrm{Ns} / \mathrm{m}^{3 / 2}\right]$

$D$ distance $[\mathrm{m}]$

e restitution coefficient $[-]$

E elasticity modulus [Pa]

$H \quad$ height $[\mathrm{m}]$

fraction $[-]$

I inertia tensor $\left[\mathrm{kgm}^{2}\right]$

$k \quad$ stiffness $\left[\mathrm{Nm}^{-3 / 2}\right]$

L Length [m]

$m \quad$ mass $[\mathrm{kg}]$

M Moment of contact force [Nm]

N normal contact force $[\mathrm{N}]$

n normal direction vector [-]

$P \quad$ probability [-]

Q $\quad$ rotation matrix [-]

$\mathbf{r} \quad$ CoM position vector $[\mathrm{m}]$

$R \quad$ radius [m]

t tangential direction vector [-]

T tangential contact force [N]

v particle CoM velocity $[\mathrm{m} / \mathrm{s}]$

$\mathbf{X}$ model parameter $[-]$

\section{Abbreviations}

\section{CoM Centre of Mass}

CSP Cylindrical Spread Pattern

DEM Discrete Element Method

IDV Initial downward velocity (out of bin)

IPA Initial Particle area (on the disc)

N/A Not available

$R D \quad$ Relative error or deviation

\section{Greek symbols}

$\alpha \quad$ segment angle in CSP $\left[{ }^{\circ}\right]$

$\beta \quad$ width of cylindrical spread pattern $\left[{ }^{\circ}\right]$

$\delta \quad$ contact virtual overlap [m]

$\Delta t \quad$ time step [s]

$\chi \quad$ eccentricity [-]

$\ell_{d} \quad$ conical disc inclination $\left[{ }^{\circ}\right]$

$\mu \quad$ friction coefficient $[-]$

$\nu \quad$ Poisson's ratio -

$\rho \quad$ density $\left[\mathrm{kg} / \mathrm{m}^{3}\right]$

$\Psi \quad$ bulk flow [kg/s]

$\omega \quad$ angular velocity of particle[rad/s]

$\Omega_{d} \quad$ disc rotational speed [rpm]

\section{Acknowledgments}

The authors are very grateful to the IWT, FWO and the K.U. Leuven Research Fund for financial support and to Amazone Werke $\mathrm{GmbH}$, Hasbargen, Germany.

\section{References}

[1] V.I. Arnold, Mathematical methods of classical mechanics, Springer Verlag, 1978. 812

[2] N.V. Brilliantov, F. Spahn, J. Hertzsch, T. Pöschel, Model for collisions in granular 813 gases, Physical Review E 53 (1996) 5382-5392.

3] N.V. Brilliantov, T. Pöschel, Rolling friction of a viscous sphere on a hard plane, 815 Europhysics Letters 42 (1998) 511-516.

[4] E. Dintwa, Development of accurate contact force models for use with Discrete 817 Element Method (DEM)The Discrete element Method (DEM) modelling of bulk 818 fruit handling processes. PhD Thesis, Faculteit Landbouwkundige en Toegepaste 819 Biologische Wetenschappen, K.U.Leuven, Belgium (2006).

[5] G. Garberoglio, Dynamical properties of H-bonded liquids: A theoretical and 821 computer simulations study. Phd Thesis. Faculty of Physical and Natural 822 Mathematical sciences, University of Trento (2001).

[6] T.E. Grift, J.W. Hofstee, Measurement of velocity and diameter of individual 824 fertilizer particles by an optical method, Journal of Agricultural Engineering 825 Research 66 (1997) 235-238.

[7] E.J. Haug, Intermediate Dynamics, Prentice Hall, Englewood Cliffs, N.J, 1992.

[8] J.W. Hofstee, Handling and spreading of fertilizers Part 5: the spinning disc type 828 fertilizer spreader, Journal of Agricultural Engineering Research 62 (1995) 829 143-162.

[9] F.M. Inns, A.R. Reece, The theory of the centrifugal distributor II: motion on the 831 disc, off-centre feed, Journal of Agricultural Engineering Research 7 (4) (1962) 832 345-353.

[10] K.L. Johnson, Contact Mechanics, Cambridge University Press, 1987.

[11] N. Mohsenin, Physical properties of plant and animal materials, Gordon and 835 Breach, 1986.

[12] H.P. Kuo, P.C. Knight, D.J. Parker, M.J. Adams, J.P.K. Seville, Discrete element 837 simulations of a high-shear mixer, Advanced Powder Technology 15 (3) (2004) 838 297-309.

[13] G. Kuwabara, K. Kono, Restitution coefficient in a collision between 2 spheres, 840 Japanese Journal of Applied Physics 26 (8) (1987) 1230-1233.

[14] B. Olieslagers, Fertilizer distribution modelling for centrifugal spreader design. 842 PhD Thesis, Faculteit Landbouwkundige en Toegepaste Biologische Wetenschap- 843 pen, K.U.Leuven, Belgium (1997).

[15] D.E. Patterson, A.R. Reece, The theory of the centrifugal distributor I: motion on the 845 disc, near-centre feed, Journal of Agricultural Engineering Research 7 (3) (1962) 846 $232-240$.

[16] E. Piron, D. Miclet, Spatial distribution measurement: a new method for the 848 evaluation and testing of centrifugal fertilizer spreaders, 2nd International 849 Symposium on Centrifugal Fertilizer Spreading, 24-25 septembre, Montoldre, 850 France, 2006.

[17] T. Pöschel, T. Schwager, Granual dynamics: Models and Algorithms, Springer, 852 Berlin, 2005.

[18] J. Reumers, E. Tijskens, H. Ramon, J. De Baerdemaeker, Experimental characterisa- 854 tion of dynamical properties of fertilizer grains in a centrifugal disk spreader: 855 towards an alternative for spreading hall measurements, Biosystems Engineering 856 86 (3) (2003) 327-337.

[19] J. Schäfer, S. Dippel, D.E. Wolf, Force Schemes in simulations of granular materials, 858 Journal de Physique (France) 6 (1996) 5-20.

[20] T. Schwager, T. Pöschel, Contact of viscoelastic spheres, in: D.E. Wolf, P. Grassberger 860 (Eds.), Friction, Arching, Contact Dynamics, World Scientific, Singapore, 1996. 861

[21] R.L. Stewart, J. Bridgwater, Y.C. Zhou, A.B. Yu, Simulated and measured flow of 862 granules in a bladed mixer-a detailed comparison, Chemical Engineering Science 863 56 (19) (2001) 5457-5471.

[22] E. Tijskens, Modelling particle shape in DEM simulations, 2nd International 865 Symposium on Centrifugal Fertilizer Spreading 24-25 septembre, Montoldre, 866 France, 2006.

[23] J. Vangeyte, B. Sonck, P. Van Liedekerke, H. Ramon. Comparison of two methods to 868 Q1 measure outlet velocity of fertilizer grains from a rotary disc. AgEng 2004869 proceeding, 336-337.

[24] P. Van Liedekerke, E. Tijskens, E. Dintwa, J. Anthonis, H. Ramon, A discrete element 871 model for simulation of a spinning disc fertilizer spreader I. Single particle 872 simulations, Powder Technology 170 (2) (2006) 71-85.

[25] Y.C. Zhou, B.D. Wright, R.Y. Yang, B.H. Xu, A.B. Yu, Rolling friction in the dynamic 874 simulation of sandpile formation, Physica. A, Statistical Mechanics and its 875 Applications 269 (2-4) (1999) 536-553.

[26] Y.C. Zhou, A.B. Yu, R.L. Stewart, J. Bridgwater, Microdynamic analysis of the particle 877 flow in a cylindrical bladed mixer, Chemical Engineering Science 59 (6) (2004) 878 1343-1364. 